\title{
WIDE-BAND PUSH-PULL AMPLIFIER FOR HIGH GRADIENT CAVITY
}

\author{
Y. Sato, M. Fujieda, Y. Mori, H. Nakayama, C. Ohmori, R. Muramatsu, \\ T. Uesugi, M. Yamamoto, M. Toda, A. Takagi and M. Yoshi, KEK; \\ Y. Taniguchi, Denki Kogyo Co.; K. 'OHTA, Ohta Electronics Co.
}

\section{Abstract}

A high gradient cavity(HGC) using high permeability magnetic alloy (MA) cores has been developed at KEK for the JHF(Japan Hadron Facility) proton synchrotrons. In this paper, we describe two types of push-pull amplifiers to drive the cavity, which were constructed for verifying the cavity performance. An accelerating voltage of $20 \mathrm{kV}$ across the gap of the HGC was generated by the high power push-pull amplifier. This is equivalent to high gradient field of $50 \mathrm{kV} / \mathrm{m}$. The other amplifier and the cavity were prepared for accelerating heavy ions at the HIMAC(Heavy Ion Medical Accelerator in Chiba). For this purpose, it is necessary to sweep the frequency over the wide range from $1 \mathrm{MHz}$ to $8 \mathrm{MHz}$.

\section{INTRODUCTION}

In order to generate a high field gradient with HGC using MA-cores, a high power push-pull type of rf amplifier which uses two $150 \mathrm{~kW}$ class tetrodes $(4 \mathrm{CW} 150,000 \mathrm{E})$ was constructed. The HGC with MA has the following two remarkable features: (1)the ability to generate high gradient field up to $50 \mathrm{kV} / \mathrm{m}$ and (2)broad band impeadance over the frequency range of $5 \mathrm{MHz}$. The characteristics of the HGC with MA have been reported [1, 2, 3, 4]. We have built in the medium power push-pull amplifier using two $30 \mathrm{~kW}$ class tetrodes $(4 \mathrm{CW} 30,000 \mathrm{~A})$ for acceleration of heavy ions at HIMAC.

\section{PUSH-PULL AMPLIFIER}

A schematic view for the RF system including the cavity and the amplifier is shown in Figure 1. The push-pull amplifier in class-B operation has the following two features: (1)The high rf output voltage close to dc plate voltage can be obtained. That is, the plate dissipation is less than that of an amplifier in class-A operation. (2)The output voltage will be less distorted even if a tuning circuit is not utilized for the load cavity. This is because the even harmonics introduced by nonlinearity of the dynamic-tube characteristics are eliminated at the output by a push-pull connection.

\section{$2.1300 k W$ Push-pull Amplifier}

Concerning the tube performance of the push-pull amplifier using two tetrodes $4 \mathrm{CW} 150,000 \mathrm{E}$, the measured and calculated specifications are presented in Table 1.

\begin{tabular}{l|c|c}
\hline \hline Parameter & $\begin{array}{c}\text { Measured } \\
\text { value }\end{array}$ & $\begin{array}{c}\text { Calculated } \\
\text { value }\end{array}$ \\
\hline DC plate voltage $V_{p}$ & $15 \mathrm{kV}$ & $15 \mathrm{kV}$ \\
DC idling current $I_{p o}$ & $2.9 \mathrm{~A}$ & $1.0 \mathrm{~A}$ \\
DC plate current $I_{p}$ & $3.71 \sim 6.84 \mathrm{~A}$ & $5.94 \mathrm{~A}$ \\
DC screen voltage $V_{g 2}$ & $1550 \mathrm{~V}$ & $1500 \mathrm{~V}$ \\
DC screen current $I_{g 2}$ & not measured & $466 \mathrm{~mA}$ \\
DC grid voltage $V_{g} 1$ & $-390 \mathrm{~V}$ & $-390 \mathrm{~V}$ \\
DC grid current $I_{g 1}$ & $65 \sim 87 \mathrm{~mA}$ & $44 \mathrm{~mA}$ \\
Driving grid voltage $E_{g}$ & $602 \mathrm{~V}$ & $602 \mathrm{~V}$ \\
Fundamental plate curr. $I_{p 1}$ & - & $25.7 \mathrm{~A}$ \\
Peak plate voltage $E_{p m}$ & $14 \mathrm{kVp}$ & $14 \mathrm{kVp}$ \\
Peak plate current $I_{p m}$ & not measured & $85 \mathrm{~A}$ \\
Plate input power $W_{i}$ & $55.7 \sim 102.6 \mathrm{~kW}$ & $89.1 \mathrm{~kW}$ \\
Plate output power $W_{o}$ & - & $59.0 \mathrm{~kW}$ \\
Plate dissipation $W_{l}$ & $55.4 \mathrm{~kW}(\mathrm{max})$. & $30.1 \mathrm{~kW}$ \\
Voltage at gap end $V_{g a p}$ & $-14 \mathrm{kVp},+7.2 \mathrm{kVp}$ & $\pm 14 \mathrm{kVp}$ \\
Accelerating voltage $V_{a c c}$ & $\pm 16 \mathrm{kVp}$ & - \\
\hline \hline
\end{tabular}

Table 1: Tube performance of the $300 \mathrm{~kW}$ push-pull amplifier.

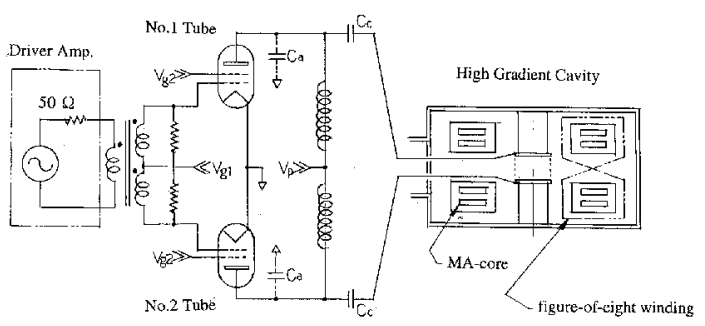

Figure 1: Schematic view for the RF system.

The measured values were taken when the amplifier was operated at $20 \%$ duty factor in burst mode sweeping the frequency from $2 \mathrm{MHz}$ to $3.4 \mathrm{MHz}$ with repetition rate of $2 \mathrm{~Hz}$. The calculated values were derived from the operating line on the constant-current curves for the tube by reading the instantaneous values of plate, screen, and grid current during the negative half cycle of the plate voltage swing. In order to compare the measured values to the calculated ones, the calculated values for $I_{p}, I_{g 2}, I_{g 1}, W_{i}, W_{o}$ and $W_{l}$ are adjusted to $20 \%$ duty factor operation. The measured value of idling current $I_{p o}$ was higher than the calculated value. This means that the amplifier was in class $\mathrm{AB}_{2}$ operation mode, where the conduction angle of plate current was more than $180^{\circ}$ and grid current was flowing. Thus the measured plate dissipation $W_{l}$ was rather higher than the calculated one. 
Since the MA-loaded HGC has a low-Q $(<1)$ value and the cavity is directly connected to the plate of the amplifier via a low impedance capacitor $\left(C_{c}\right)$ as shown in Figure 1, the plate voltage swing would substantially depend on the cavity impedance. The cavity impedance can be estimated by dividing the peak plate voltage by the peak plate current $: E_{p m} / I_{p m}=165 \Omega$. This would be consistent with the cavity impedance of approx. $178 \Omega$ at $2.3 \mathrm{MHz}$ which was measured from the plate by a network analyzer.

Since the rf plate current at a positive rf half cycle is nearly zero, the power delivered by one tube is about a half of $W_{o}$, which is given by $W_{o}=E_{p m} \cdot I_{p 1} / 2$, Thus the power $W_{o}$ is almost the output power delivered by two tubes. Using the values of $E_{p m}$ and $I_{p 1}$ presented in Table 1 , the power $W_{o}$ becomes $295 \mathrm{~kW}$ in cw mode operation. Taking account of rather high dc screen current $I_{g 2}$ in Table 1, we should be carefull that the screen dissipation can not exceed the normal rating of the tube. It would be pointed out that the excessive driving power at the control grid should be avoided because it may allow instantaneous plate voltage drop lower than the dc screen voltage, resulting in abnormal screen dissipation. In addition, if the dc plate voltage can be raised, it may contribute not only to decrease screen dissipation but to reduce the distortion of the accelerating voltage waveform, which may be introduced by plate current saturation.

\subsection{0kW Push-Pull Amplifier}

The MA-loaded HGC and the $60 \mathrm{~kW}$ push-pull amplifier with two tetrodes 4CW30,000A were installed at HIMAC. In order to accelerate heavy ions at HIMAC, it is necessary to sweep the frequency over the wide range from $1 \mathrm{MHz}$ to $8 \mathrm{MHz}$. The accelerating rf voltage depends not only on the characteristics of the shunt impedance, but also the gainfrequency characteristics of the amplifier. With respect to the $60 \mathrm{~kW}$ push-pull amplifier, a maximum gain drop at the grid input circuit was $4.6 \mathrm{~dB}$ at the frequency range from $1 \mathrm{MHz}$ to $8 \mathrm{MHz}$. Thus proper voltage control was employed to obtain the constant rf voltage $[3,5,6]$.

\section{EQUIVALENT CIRCUIT AND COUPLING METHOD}

\subsection{Equivalent Circuit for the RF system}

The impedance seen from the tube was measured by a network analyzer. It includes both impedance of the cavity and the plate circuit of the amplifier. The Figure 2 shows the measured impedance where the fundamental resonance was stayed at $2.3 \mathrm{MHz}$, on the other hand, series resonance was at $14.8 \mathrm{MHz}$ and parallel resonance was at $21.5 \mathrm{MHz}$, respectively. The impedance is almost equal to the shunt impedance $Z_{s}$ corresponding to one-half of the cavity at low frequency region below $5 \mathrm{MHz}$, even though two-halves of the cavity are coupled each other with the capacitance $C_{g}$ for the accelerating gap. This would be justified by a comparison : $\left|1 / \omega C_{g}\right| \gg\left|Z_{s}\right|$, where $\left|1 / \omega C_{g}\right| \cong$
$3.8 k \Omega ;\left|Z_{s}\right| \leq 178 \Omega$ around the frequency of $2.3 \mathrm{MHz}$. The value of $Z_{s}$ can be estimated by the parameters $L_{p}, C_{p}$ and $R_{p}$ of the equivalent circuit shown in Figure 3 . The shunt resistance $R_{p}$ at fundamental resonance is $178 \Omega$ as abovementioned. On the other hand, the impedance seen from the both ends of the gap is almost twice the $R_{p}$ which is reported by the referenced paper [3]. It would be explained as the impedance quite corresponds to that of two-halves of the cavity.

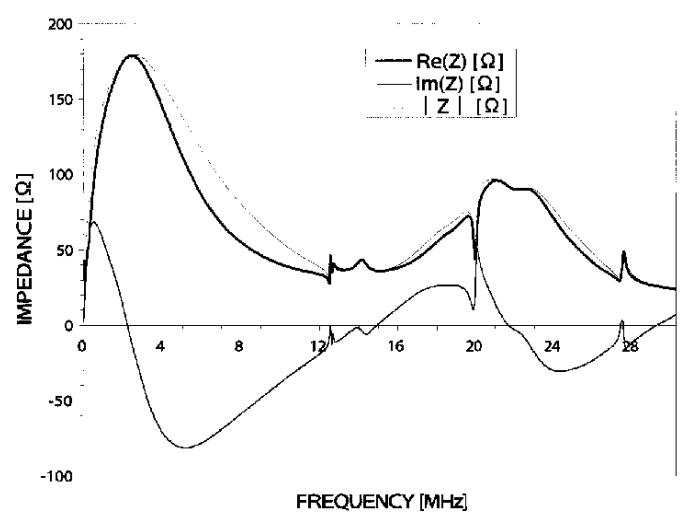

Figure 2: Impedance characteristics seen from the plate of one tube.

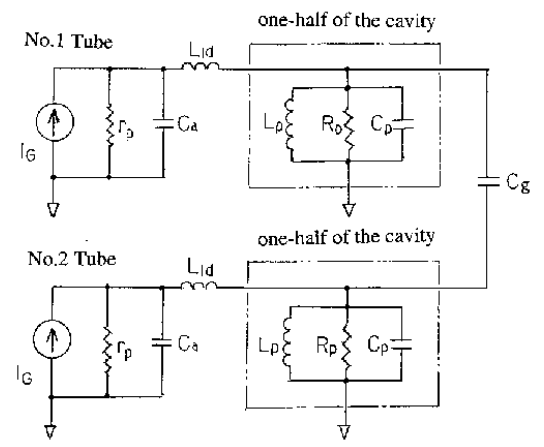

$$
\begin{aligned}
& \text { Zs: Shunt impedance corresponding to one-halt of the cavity } \\
& \frac{1}{Z_{s}}=\frac{1}{R_{p}}+j\left(\omega C_{p}-\frac{1}{\omega L_{p}}\right) \\
& R_{p}: \text { Shunt resistance of the cavity } \\
& \mathrm{L}_{\mathrm{p}} \text { : Parallel inductance of the cavity } \\
& C_{\mathrm{p}} \text { : Parallel capacitance of the cavity } \\
& \mathrm{C}_{g} \text { : Capacitance of the accelerating gap } \\
& \text { Ld: Lead inductance of the conductor } \\
& \text { between the tube plate and the cavity } \\
& \text { Ca: output capacilance of the tube } \\
& \text { rp: Plate internal resistance } \\
& \text { IG: Current source generated by the amplifier }
\end{aligned}
$$

Figure 3: Equivalent circuit for the RF system.

\subsection{Coupling Method with Figure-of-Eight Winding}

By means of coupling two-halves of the cavity with a figure-of-eight winding looped through each other, the RF cavity is intrinsically capable of operating in a push pull mode [7]. Due to this coupling a load impedance seen 
from the plate substantially becomes one half of the shunt impedance $Z_{s}$. The figure-of-eight winding works as a transformer as shown in Figure 5. The characteristics of load impedance for the cavity with this coupling is also represented in Figure 4. The load impedance which is one half of the $Z_{s}$ may cause a plate dissipation to increase, because it may deteriorate the matching condition of the impedance between the plate and the cavity.

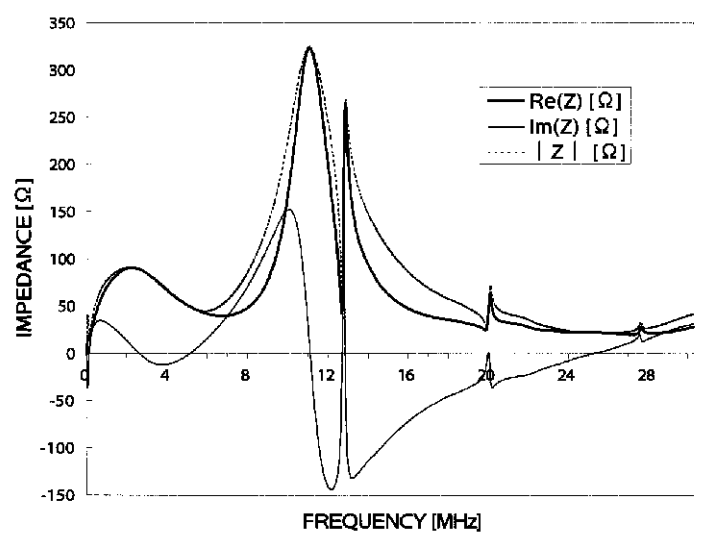

Figure 4: Impedance characteristics of the cavity seen from the plate of one tube; Two halves of each cavity are looped with a figure-of-eight winding.

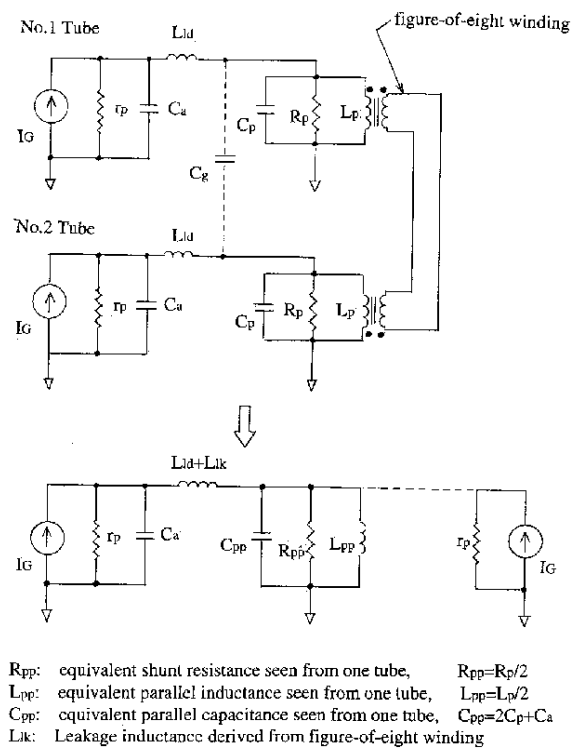

Figure 5: Equivalent circuit for two-halves of the cavity coupled with a figure-of-eight winding.

A parasitic resonance at the frequency around $11 \mathrm{MHz}$ can cause harmful distortion to the accelerating voltage. The circuit with this coupling can be simplified as a reactance circuit shown in Figure 6. Applying a same reactance function described in the previous paper [8], parallel resonant frequency $f_{h}$ will be given by the following equation.

$$
f_{h}=1 / 2 \pi \sqrt{L_{2}\left(\frac{C_{1} C_{2}}{C_{1}+C_{2}}\right)}
$$

In the equation, $L_{2}$ comprises leakage inductance $L_{l k}$ and lead inductance $L_{l d}, C_{2}$ is the tube capacitance and $C_{1}$ is the sum of the tube capacitance and total capacitance for two-halves of the cavity. Assuming parameters as shown in Figure $6, f_{h}$ is calculated to be about $13.5 \mathrm{MHz}$, which is not so large different from the measured value of $11.0 \mathrm{MHz}$. The figure-of-eight winding intrinsically introduces a parasitic resonance and allows its frequency lower. We did not adopt the figure-of-eight winding.
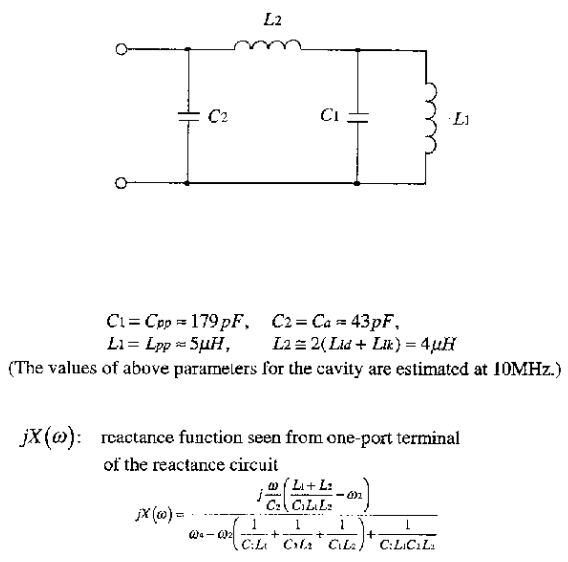

Figure 6: Reactance circuit.

\section{CONCLUSION}

A push-pull amplifiers using two tetrodes 4CW150,000E has delivered the output power of $295 \mathrm{~kW}$ in cw mode to generate an accelerating voltage of about $16 \mathrm{kV}$ in the HGC. The maximum voltage of $20 \mathrm{kV}$ was achieved at a small duty factor operation. Another amplifier using two tetrodes $4 \mathrm{CW} 30,000 \mathrm{~A}$ has generated an rf voltage of about $4 \mathrm{kV}$ at $3 \mathrm{MHz}$ when sweeping the frequency from $1 \mathrm{MHz}$ to $8 \mathrm{MHz}$. Further investigation and development to improve the gainfrequency characteristics of grid input circuit are going to be carried out.

We have investigated a figure-of-eight winding coupling. We found that the impedance seen from the tube drops to a quarter of the total cavity shunt impedance and a parasitic resonance is inevitably introduced.

\section{REFERENCES}

[1] Y. Mori et al., Proc. of EPAC98, p1796, Stockholm,(1998)

[2] Y. Mori et al.; "A new type of rf cavity for high intensity proton synchrotron", Proc. of Symp. On ACC. Sci. and Tech., Nishiharima, Japan, 1997

[3] C. Ohmori et al., in this conference

[4] M. Fujieda et al., Proc. of f APAC98, Tukuba,(1998)

[5] M. Yamamoto et al., in this conference

[6] R. Muramatsu et al., in this conference

[7] M. Meth et al.; "PUSH PULL OPERATION OF THE RF CAVITY”, Booster Tec. Note No.84, B.N.L., 1987

[8] Y. Sato et al., Proc. of APAC98, Tukuba,(1998) 